\title{
Comparative study of Closed loop and Open loop Contrast Enhancement Techniques
}

\author{
Ramkumar. M. U, Kavitha. N. Nair, \\ $P G$ (M.Tech) Scholar, Department of ECE, MG University College of Engineering, Thodupuzha Kerala \\ Asst. Professor, Department of ECE ,MG University College of Engineering, Thodupuzha, Kerala - 685587
}

\begin{abstract}
The remote sensing images played an important role in all fields such as agriculture, meteorology, geology, education etc. As the rising demand for high quality remote sensing images, contrast enhancement techniques are required for better visual perception and color reproduction. In this paper we explained a comparative study on open loop and closed loop contrast enhancement techniques which use dominant brightness level analysis and adaptive intensity transformation with discrete wavelet transform. Although various histogram equalization methods are proposed in the literature. They tend to degrade the overall image quality by exhibiting saturation artifacts in both low- and high-intensity layers. The proposed algorithms overcome this problem. Here in open lop system DWT is performed first and then decompose the LL subband into low- middle-high- intensity layers using log-average luminance. Intensity layer transfer functions are adaptively estimated by using knee transfer function and the gamma adjustment function based on dominant brightness level on each layer. After the intensity transformation the enhance image is get back by taking Inverse DWT. We can add a feedback system for better contrast enhancement by providing a set value of performance measure usually EME for better result. The performances of two approaches are evaluated with parameters such Mean Square Error (MSE), Measure of Enhancement (EME), Peak Signal to Noise Ratio (PSNR) and Mean Absolute Error (MAE).
\end{abstract}

Index Terms: Adaptive intensity transfer function, closed loop, contrast enhancement, discrete wavelet Trans form (DWT), dominant brightness level analysis, open loop, remote sensing.

\section{Introduction}

The quality of an image as perceived by a human can be improved by Image enhancement techniques. The aim of image enhancement is to improve the interpretability or perception of information in images for human viewers, or to provide better input for other automated image processing techniques. These techniques are most useful because many satellite images when examined on a colour display give inadequate information for image interpretation. There is no conscious effort to improve the fidelity of the image with regard to some ideal form of the image. There exists a wide variety of techniques for improving image quality. The contrast stretch, density slicing, edge enhancement, and spatial filtering are the more commonly used techniques. Image enhancement is attempted after the image is corrected for geometric and radiometric distortions. The proposed paper introduces some new methods for contrast enhancement.

Histogram equalization (HE) [1] has been the most popular approach to enhancing the contrast in various application areas such as medical image processing, object tracking, speech recognition, etc. HE-based methods cannot, however, maintain average brightness level, which may result in either under- or oversaturation in the processed image. For overcoming these problems, bi-histogram equalization (BHE) [2] method have been proposed by using decomposition of two subhistograms. For further improvement, the recursive mean-separate HE (RMSHE) [3] method iteratively performs the BHE and produces separately equalized subhistograms. However, the optimal contrast enhancement cannot be achieved since iterations converge to null processing. And also proposed a modified HE method which is based on the singular-value decomposition of the LL subband of the discrete wavelet transform (DWT) [4] [5] . In spite of the improved contrast of the image, this method tends to distort image details in low- and high-intensity regions.

In remote sensing images, the common artifacts caused by existing contrast enhancement methods, such as drifting brightness, saturation, and distorted details; need to be minimized because pieces of important information are widespread throughout the image in the sense of both spatial locations and intensity levels. For this reason, enhancement algorithms for satellite images not only improve the contrast but also minimize pixel distortion in the low- and high-intensity regions.

For achieving this goal we presented a new method which uses dominant brightness level analysis, adaptive intensity transfer function [6], along with DWT [7]. We applied both the open loop and closed loop techniques for comparative study. 


\section{Image Decomposition-Discrete Wavelet Transform (DWT)}

An image is represented as a two dimensional array of coefficients, each coefficient representing the brightness level in that point. When looking from a higher perspective, the coefficients cannot be differentiated as more important one, and lesser important one. But most natural images have smooth colour variations, with the fine details being represented as sharp edges in between the smooth variations. Technically, the smooth variations in colour can be termed as low frequency variations and the sharp variations as high frequency variations. The low frequency components constitute the base of an image and the high frequency components add upon them to refine the image thereby giving a detailed image. Hence the smooth variations are demanding more importance than the details. Separating the smooth variations and details of the image can be done in many ways. One such way is the decomposition of the image using Discrete Wavelet Transform (DWT)

DWT decomposes an image into four sub-bands: approximation and detailed sub-bands- horizontal, vertical, and diagonal. The detailed sub-bands shows variations along the columns (horizontal edges), rows (vertical edges), and diagonals (diagonal edges) respectively As shown in Fig. 1 at each level, approximation sub-band is decomposed into the above mentioned four sub-bands. A low pass filter and a high pass filter are chosen, such that they exactly have the frequency range between themselves. The filter pair is called the analysis filter pair. First the low pass filter is applied for each row of data, thereby getting the low frequency components of the row. Now the high pass filter is applied for the same row of data, and similarly the high pass components are separated and placed by the side of the low pass components. This procedure is done for all rows. Next, the filtering is done for each column of the intermediate data. The resulting two dimensional arrays of coefficients contain four bands of data, each labeled as LL (low- Low), HL (high-low), LH (Low-High) and HH (HighHigh). The LL band can be decomposed once again in the same manner, thereby producing even more subbands. This can be done up to any level, thereby resulting in a pyramidal decomposition as shown in Fig.1. The LL band at the highest level can be classified as most important and the other detail bands can be classified as of lesser importance, with the degree of importance decreasing from the top of the pyramid to the bands at the bottom. In Fig. 1 three level decomposition is shown. Here, L \& H represents low frequency and high frequency components respectively. The sub-band LL denotes the low frequency component of the image, which is the approximation sub-band of the original image. The sub-band HL is the low frequency component in horizontal direction and the high frequency component in vertical direction, which shows the horizontal edge in the original image. The sub-band LH is the high frequency component in horizontal direction and the low frequency component in vertical direction, which shows the vertical edge in the original image. The sub-band HH is the high frequency component, which manifests the diagonal edges in the original image.

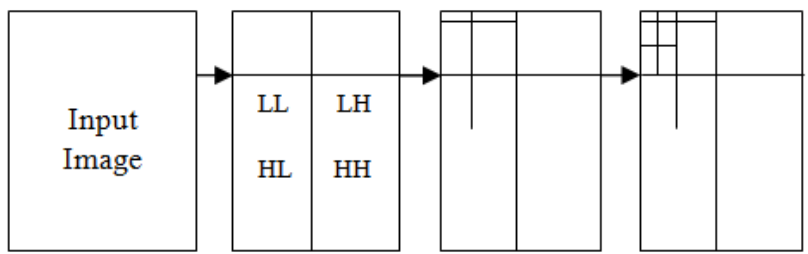

Fig. 1. Flow chart of the DWT decomposition

\section{Analysis Of Dominant Brightness Levels}

If we do not consider spatially varying intensity distributions, the correspondingly contrast enhanced images may have intensity distortion and lose image details in some regions. For overcoming these problems, first decompose the input image into multiple layers of single dominant brightness levels. To use the lowfrequency luminance components, perform the DWT on the input remote sensing image and then estimate the dominant brightness level using the log average luminance in the LL subband. Since high-intensity values are dominant in the bright region, and vice versa, the dominant brightness [12] [13] at the position ( $x, y)$ is computed as,

$$
\mathrm{D}(\mathrm{x}, \mathrm{y})=\exp \left(\frac{1}{\mathrm{~N}_{\mathrm{L}}} \sum_{(\mathrm{x}, \mathrm{y}) \mathrm{\varepsilon S}}\{\log \mathrm{L}(\mathrm{x}, \mathrm{y})+\varepsilon\}\right)
$$

Where $S$ represents a rectangular region encompassing $(x, y), L(x, y)$ represents the pixel intensity at $(\mathrm{x}, \mathrm{y}), \mathrm{N}_{\mathrm{L}}$ represents the total number of pixels in $\mathrm{S}$, and $\varepsilon$ represents a sufficiently small constant that prevents the log function from diverging to negative infinity. The low-intensity layer has the dominant brightness lower than the pre specified low bound. The high intensity layer is determined in the similar manner with the pre specified high bound, and the middle-intensity layer has the dominant brightness in between low and high bounds. The normalized dominant brightness varies from zero to one, and it is practically in the range between 
0.5 and 0.6 in most images. For safely including the practical range of dominant brightness, we used 0.4 and 0.7 for the low and high bounds, respectively.

\section{Edge Preserving Contrast Enhancement Using Adaptive Intensity Transformation}

Based on the dominant brightness in each decomposed layer, the adaptive intensity transfer function is generated. Since remote sensing images have spatially varying intensity distributions, we estimate the optimal transfer function in each brightness range for adaptive contrast enhancement. The adaptive transfer function is estimated by using the knee transfer and the gamma adjustment functions. For the global contrast enhancement, the knee transfer function stretches the low-intensity range by determining knee points according to the dominant brightness of each layer. More specifically, in the low-intensity layer, a single knee point is computed as,

$$
\mathrm{P}_{1}=\mathrm{b}_{1}+\mathrm{w}_{\mathrm{l}}\left(\mathrm{b}_{1}-\mathrm{m}_{\mathrm{l}}\right)
$$

Where $b_{l}$ represents the low bound, $w_{l}$ represents the tuning parameter, and $m_{l}$ represents the mean of brightness in the low intensity layer. For the high-intensity layer, the corresponding knee point is computed as,

$$
\mathrm{P}_{\mathrm{h}}=\mathrm{b}_{\mathrm{h}}-\mathrm{w}_{\mathrm{h}}\left(\mathrm{b}_{\mathrm{h}}-\mathrm{m}_{\mathrm{h}}\right)
$$

Where $b_{h}$ represents the high bound, $w_{h}$ represents the tuning parameter, and $m_{h}$ represents the mean of brightness in the low intensity layer. In the middle intensity layer, two knee points are computed as,

$$
\begin{gathered}
P_{m l}=b_{l}-w_{m}\left(b_{m l}-m_{m}\right)+\left(p_{l}-p_{h}\right) \\
P_{m h}=b_{h}+w_{m}\left(b_{m h}-m_{m}\right)+\left(p_{l}-p_{h}\right)
\end{gathered}
$$

Where $w_{m}$ represents the tuning parameter and $m_{m}$ represents the mean brightness in the middleintensity layer.

The global image contrast is determined by tuning parameter $w_{i}$ for i $\epsilon\{l, m, h\}$. Although the contrast is more enhanced as the $w_{i}$ increases, the resulting image is saturated and contains intensity discontinuity. Here adjust only the middle-intensity tuning parameter wm for reducing such artifacts. Since the knee transfer function tends to distort image details in the low- and high intensity layers, additional compensations performed using the gamma adjustment function. The gamma adjustment function is modified from the original version by scaling and translation to incorporate the knee transfer function as,

$$
G_{k}(L)=\left\{\left(\frac{L}{M_{k}}\right)^{1 / \gamma}-\left(1-\frac{L}{M_{k}}\right)^{1 / \gamma}+1\right\}
$$

For $\mathrm{k} \epsilon\{l, m, h\}$ Where $\mathrm{M}$ represents the size of each section intensity range, such as $M_{l}=b_{l}, M_{m}=$ $b_{h}-b_{l}$ and $M_{h}=1-b_{h}, \mathrm{~L}$ represents the intensity value, and $\gamma$ represents the pre specified constant. The pre specified constant $\gamma$ can be used to adjust the local image contrast. As $\gamma$ increases, the resulting image is saturated around $b_{l} / 2, b_{h}-b_{l} / 2$ and $1-b_{h} / 2$.Therefore, the $\gamma$ value is selected by computing maximum values of adaptive transfer function in ranges $\left\{0 \leq \mathrm{L}<\mathrm{b}_{\mathrm{l}} / 2\right\},\left\{\mathrm{b}_{\mathrm{l}} \leq \mathrm{L}<\left(\mathrm{b}_{\mathrm{h}}-\mathrm{b}_{\mathrm{l}} / 2\right)\right\}$ and $\left\{\mathrm{b}_{\mathrm{h}} \leq \mathrm{L}<(1-\right.$ $\left.\left.\mathrm{b}_{\mathrm{h}} / 2\right)\right\}$ which are smaller than, $\mathrm{b}_{\mathrm{l}} / 2, \mathrm{~b}_{\mathrm{h}}-\mathrm{b}_{\mathrm{l}} / 2$, and $1-\mathrm{b}_{\mathrm{h}} / 2$ respectively.

The proposed adaptive transfer function is obtained by combining the knee transfer function and the modified gamma adjustment function. Three intensity transformed layers by using the adaptive intensity transfer function are fused to make the resulting contrast-enhanced image in the wavelet domain. Extract most significant two bits from the low-, middle-, and high-intensity layers for generating the weighting map, and compute the sum of the two bit values in each layer and select two weighting maps that have two largest sums. For removing the unnatural borders of fusion, weighting maps are employed with the Gaussian boundary smoothing filter. As a result, the fused image $\mathrm{F}$ is estimated as,

$$
\mathrm{F}=\mathrm{W}_{1} \times \mathrm{c}_{1}+\left(1-\mathrm{W}_{\mathrm{l}}\right) \times\left\{\mathrm{W}_{2} \times \mathrm{c}_{\mathrm{m}}+\left(1-\mathrm{W}_{2}\right) \times \mathrm{c}_{\mathrm{h}}\right\}
$$

Where $\mathrm{W}_{1}$ represents the largest weighting map, $\mathrm{W}_{2}$ represents the second largest weighting map, $\mathrm{c}_{1}$ represents the contrast enhanced brightness in the low-intensity layer, $c_{m}$ represents the contrast-enhanced 
brightness in the middle-intensity layer, and $c_{\mathrm{h}}$ represents the contrast enhanced brightness in the high-intensity layer. Since Eqn. (7) represents the point operation, the pixel coordinate (x, y) is omitted. The fused LL subband undergoes the IDWT together with the unprocessed HL, LH, and HH subbands to reconstruct the finally enhanced image.

\section{Open Loop Contrast Enhancemnet Technique}

In this section we present a novel contrast enhancement algorithm for remote sensing images using dominant brightness level-based adaptive intensity transformation as shown in Fig.3.We can use DWT for image decomposition. This decomposes the input image into wavelet subbands and decomposes the LL subband into low, middle, and high-intensity layers by analyzing the log-average luminance of the corresponding layer. The adaptive intensity transfer functions are computed by combining the knee transfer function [8] and the gamma adjustment function [9] [10]. All the contrast enhanced layers are fused with an appropriate smoothing, and the processed LL band undergoes inverse transform together with unprocessed LH, HL, and HH subbands to reconstruct the finally enhanced image.

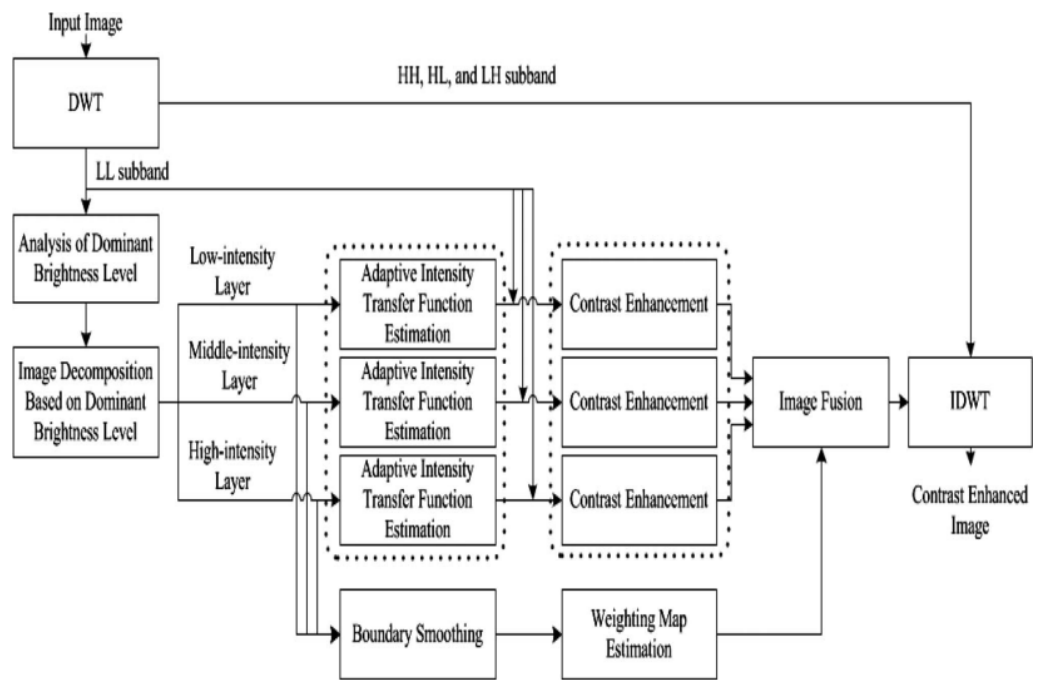

Fig.3: Block diagram of Open loop contrast enhancement technique

\section{Closed Loop Contrast Enhancemnet Technique}

In this section we present a new contrast enhancement algorithm for remote sensing images using dominant brightness level- adaptive intensity transformation as a closed loop system as shown in Fig.4.We can use DWT for image decomposition. This decomposes the input image into wavelet subbands and decomposes the LL subband into low, middle, and high-intensity layers by analyzing the log-average luminance of the corresponding layer. The knee and gamma functions are adaptively estimated and contrast enhancement is done. All the contrast enhanced layers are fused with an appropriate smoothing, and the processed LL band undergoes Inverse transform together with unprocessed $\mathrm{LH}, \mathrm{HL}$, and $\mathrm{HH}$ subbands to reconstruct the finally enhanced image.

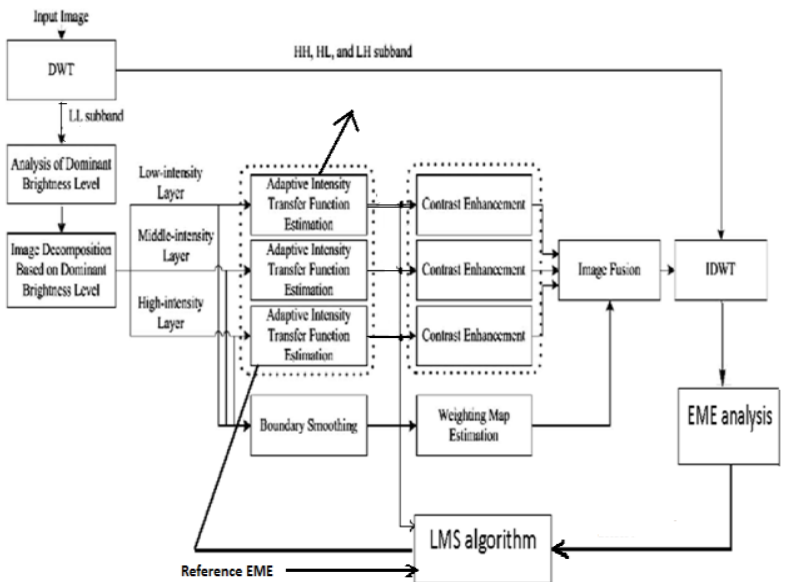

Figure 4: Block diagram of closed loop contrast enhancement technique 
In the open loop method the knee point of low, middle and high intensity layers are getting adapted. The major drawback open loop system is that less accurate and less sensitivity. In the open loop system there is no criteria for adapting the knee point. User determines the value of the tuning parameter $\mathrm{w}_{\mathrm{l}}, \mathrm{w}_{\mathrm{h}}$ and $\mathrm{w}_{\mathrm{m}}$ according to requirements. The performance of the open loop system evaluated through the parameter measure of enhancement (EME) [11] and is given by the equation (9). If the EME value of the output image is high contrast is more enhanced and vice versa. Closed loop adaptation involves making measurements of inputs or environmental characteristics, Applying this information to a computational algorithm (adaptive algorithm) and knowledge of their outcome in order to optimize a measured system performance. It is an adaptation by performance feedback. System reliability can be improved by the use of performance feedback. Different types of adaptive algorithms can be used for the adaptation. One of the most commonly used algorithms is least mean square (LMS) algorithm. Because it is used for descending on the performance surface [12] [13] and it is valid for adaptive linear combiner. Here we take knee point as the cost function. In the adaptive process with performance feedback the weight vector of the linear combiner is adjusted to cause the output EME to agree as close as possible with a desired EME. This is done by comparing the output with the desired response to obtain an error signal and then adjusting or optimizing the weight vector to minimize this error signal. The weight adaption for LMS algorithm is given by the equation (8). Where $\mathrm{X}_{\mathrm{k}}$ is the input vector, $\mathrm{W}_{\mathrm{k}}$ is the weight vector and $\mu_{\mathrm{k}}$ is the learning rate of the LMS algorithm.

$$
\mathrm{W}_{\mathrm{k}+1}=\mathrm{W}_{\mathrm{k}}+2 \mu_{\mathrm{k}} \mathrm{X}_{\mathrm{k}}
$$

\section{Experimental Results}

For evaluating the performance of the proposed algorithm, we tested low-contrast remote sensing images as shown in Fig. 6. The performance [14] of the proposed algorithms is compared with existing wellknown algorithms including standard HE, BHE, RMSHE and DWT-SVD Methods.

The proposed method is evaluated using the Peak Signal to Noise Ratio (PSNR), Mean Square Error (MSR), Measure of Enhancement (EME), Mean Absolute Error (MAE). PSNR is the quality measurement between the original image and the reconstructed image which is calculated through the Mean Squared Error (MSE). The MSE represents the cumulative squared error between the compressed and the original image, whereas PSNR represents a measure of the peak error. The EME represents the overall image quality enhanced with preserving the average brightness level and edge details in all intensity ranges and MAE represents mean of the difference existing between two images. These parameters are calculated as follows:

$$
\mathrm{EME}=\frac{1}{\mathrm{k} 1 \mathrm{k} 2} \sum_{\mathrm{i}=1}^{\mathrm{k} 2} \sum_{\mathrm{k}=1}^{\mathrm{k} 1} \frac{\mathrm{I}_{\max }(\mathrm{k}, \mathrm{l})}{\mathrm{I}_{\min }(\mathrm{k}, \mathrm{l})} \ln \frac{\mathrm{I}_{\max }(\mathrm{k}, \mathrm{l})}{\mathrm{I}_{\min }(\mathrm{k}, \mathrm{l})+\mathrm{c}}
$$

Where $\mathrm{k} 1, \mathrm{k} 2$ represents the total number of blocks in an image, $\mathrm{I}_{\max }(\mathrm{k}, \mathrm{l})$ represents the maximum value of the block, $I_{\min }(\mathrm{k}, \mathrm{l}$ represents the minimum value of the block, and $\mathrm{c}$ represents a small constant to avoid dividing by zero.

$$
\begin{gathered}
\mathrm{MSE}=\frac{1}{\mathrm{MN}} \sum_{\mathrm{i}=0}^{\mathrm{M}-1} \sum_{\mathrm{j}=0}^{\mathrm{N}-1}[\mathrm{I}(\mathrm{i}, \mathrm{j})-\mathrm{K}(\mathrm{i}, \mathrm{j})]^{2} \\
\mathrm{MAE}=\frac{1}{\mathrm{MN}} \sum_{\mathrm{i}=0}^{\mathrm{M}-1} \sum_{\mathrm{j}=0}^{\mathrm{N}-1}|\mathrm{I}(\mathrm{i}, \mathrm{j})-\mathrm{K}(\mathrm{i}, \mathrm{j})| \\
\mathrm{PSNR}=10 \log \frac{25^{2}}{\mathrm{MSE}}
\end{gathered}
$$

Where $I(i, j)$ is the input image and $K(i, j)$ is the output image. The development of the system includes the generation the codes for each of the above steps and finally we can measure the performance of the proposed method and we can do a comparison with the older methods for better distinguishing. The performance measure includes MSE, MAE, PSNR and EME. For contrast enhancement purposes the better method for evaluating the performance is EME that is the measure of enhancement.

For the experiment, we used $\gamma=1.4, \quad b_{1}=0.4$, and $b_{h}=0.7$. For three different intensity layers, $w_{l}=$ $1, \mathrm{w}_{\mathrm{m}}=3$, and $\mathrm{w}_{\mathrm{h}}=1$ were used. The results of the standard HE method show under- or oversaturation artifacts because it cannot maintain the average brightness level. Although RMSHE and GC-CHE methods can preserve the average brightness level, and better enhance overall image quality, they lost edge details in lowand high-intensity ranges On the other hand, Demirels method could not sufficiently enhance the low-intensity range because of the singular-value constraint of the target image. Fig.5 (a) - (e) [15] shows the results of the 
proposed contrast enhancement method. The overall image quality is significantly enhanced with preserving the average brightness level and edge details in all intensity rangesFig.6 shows the results of enhancement using open loop technique and Fig.7 shows result using closed loop technique. Which have significant change in EME values as shown Table 1. We also include the value of PSNR, MAE and MSE. Comparison of EME values show that the proposed Method outperforms existing enhancement methods.
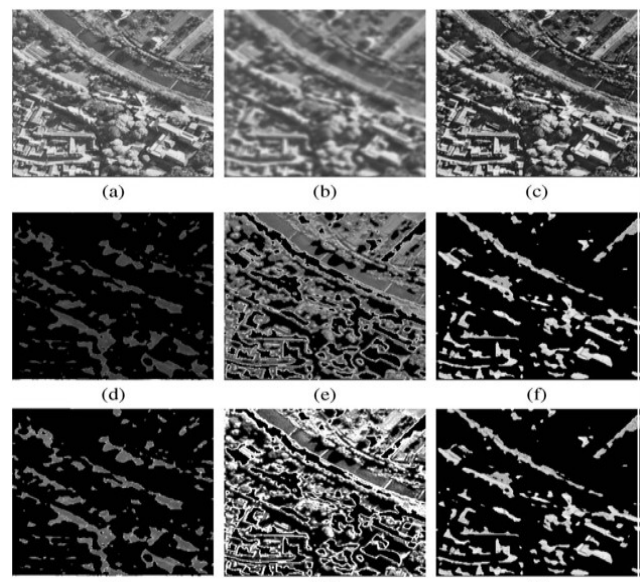

(g)

(h)

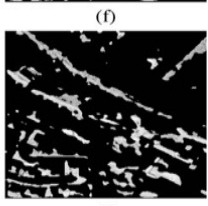

Fig. 5: Image decomposition based on the dominant brightness levels and contrast enhancement results. (a) Original image. (b) Dominant intensity analysis.(c) Enhanced result image. (d-f) Low-, middle-, and highintensity layers. (g-i) Enhanced low-, middle-, and high intensity layers.

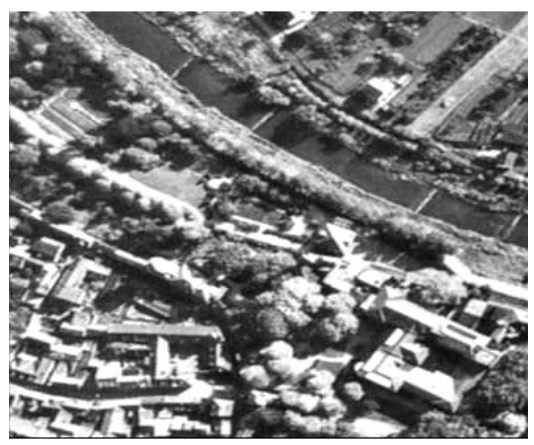

Fig.6: Enhanced image using open loop technique

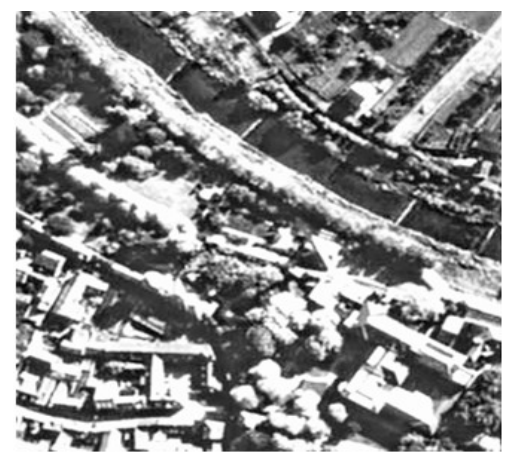

Fig.7: Enhanced image using closed loop technique

Table 1: Comparison Of Results Between Proposed Methods And Existing Methods

\begin{tabular}{|l|l|l|l|l|}
\hline \multicolumn{1}{|c|}{ Method } & EME & MSE & PSNR & MAE \\
\hline Closed loop technique & 3.2683 & 21.13 & 19.12 & 24.13 \\
\hline Open loop technique & 0.7313 & 24.65 & 20.32 & 24.23 \\
\hline SVD-DWT & 0.626 & 42.05 & 15.66 & 35.70 \\
\hline RMSHE & 0.680 & 64.31 & 10.12 & 54.06 \\
\hline BHE & 0.690 & 63.17 & 12.15 & 52.34 \\
\hline HE & 0.689 & 42.17 & 15.54 & 37.92 \\
\hline
\end{tabular}




\section{Conclusion}

In this paper we have presented a comparative study on open loop and closed loop contrast enhancement method for remote sensing images. The algorithm decomposes the input image into four wavelet subbands and decomposes the LL subband into low-, middle-, and high-intensity layers by analyzing the logaverage luminance. The adaptive intensity transfer functions are computed by combining the knee transfer function and the gamma adjustment function. All the contrast enhanced layers are fused with an appropriate smoothing, and the processed LL band undergoes the IDWT together with unprocessed, HL, LH and HH subbands. This method utilizes DWT for image decomposition in an open loop manner. To improve the image quality we use a closed loop technique. Which utilizes LMS algorithm. Using this, it can effectively enhance the overall quality and visibility of local details better than existing state-of-the-art methods including HE, BHE and RMSHE. Experimental results demonstrate that the proposed algorithms can enhance the low contrast satellite images and is suitable for various imaging devices such as consumer camcorders, real-time 3-D reconstruction systems, and computational cameras.

\section{References}

[1] R. Gonzalez and R.Woods, Digital Image Processing, 3rd ed. Englewood Cliffs, NJ: Prentice-Hall, 2007.

[2] Y. Kim, Contrast enhancement using brightness preserving bi-histogram equalization, IEEE Trans. Consum. Electron., vol. 43, no. 1, pp. 18, Feb. 1997

[3] S. Chen and A. Ramli, Contrast enhancement using recursive mean separate histogram equalization for scalable brightness preservation, IEEE Trans. Consum. Electron. vol. 49, no. 4, pp. 13011309, Nov. 2003.

[4] H. Demirel, C. Ozcinar, and G. Anbarjafari, Satellite image contrast enhancement using discrete wavelet transform and singular value decomposition, IEEE Geosci. Reomte Sens. Lett., vol. 7, no. 2, pp. 3333337, Apr. 2010.

[5] H. Demirel, G. Anbarjafari, and M. Jahromi, Image equalization based on singular value decomposition, in Proc. 23rd IEEE Int. Symp. Comput. Inf. Sci., Istanbul, Turkey, Oct. 2008, pp. 15.

[6] Eunsung Lee, Sangjin Kim, Wonseok Kang, Doochun Seo, and Joonki Paik, Contrast Enhancement Using Dominant Brightness Level Analysis and Adaptive Intensity Transformation for Remote Sensing Images, IEEE Transactions on Geosciences and remote sensing letters, vol.10,no.1,jan 2013

[7] G.Veena, V.Uma, Ch. Ganapathy Reddy, Contrast Enhancement for Remote Sensing Images with Discrete Wavelet Transform, International Journal of Recent Technology and Engineering (IJRTE) ISSN: 2277- 3878, Volume-2, Issue-3, July 2013.

[8] Y.Monobe,H.Yamashita, T.Kurosawa and H.Kotera, Dynamic range compression preserving local image contarst for digital video camera, IEEE Trans.Consum.Electron.Vol.51 ,no.1.pp.110,Feb.2005.

[9] S. Lee, An efficient contrast-based image enhancement in the compressed domain using retinex theory, IEEE Trans. Circuit Syst. Video Technol., vol. 17, no. 2, pp. 199213, Feb. 2007.

[10] W. Ke, C. Chen, and C. Chiu, BiTA/SWCE: Image enhancement with bilateral tone adjustment and saliency weighted contrast enhancement, IEEE Trans. Circuit Syst. Video Technol., vol. 21, no. 3, pp. 360364, Mar. 2010.

[11] S. S. Agaian, B. Silver, and K. A. Panetta, Transform coeffcient histogram- based image enhancement algorithms using contrast entropy, IEEE Trans. Image Process., vol. IP-16, no. 3, pp. 741758, Mar. 2007

[12] A text book of "adaptive signal processing" by Widrow and Stearns, Pearson Edition 2005

[13] Text book of "Statistical and Adaptive signal processing" by Manalokis, Ingle and Kogon, Artech House INC., 2005.

[14] S. Kim, W. Kang, E. Lee, and J. Paik, Wavelet-domain color image enhancement using filtered directional bases and frequencyadaptive shrinkage, IEEE Trans. Consum. Electron., vol. 56, no. 2, pp. 1063 1070, May 2010.

[15] Computer Vision Group-University of Granada (CVG-UGR) Image Database. [Online]. Available: http://decsai.ugr.es/cvg/dbimagenes.

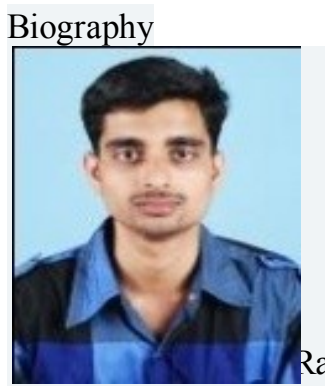

Ramkumar.M.U received his Bachelor's degree in Electronics and Instrumentation Engineering from College of Engineering, Vadakara, Kozhikod,Kerala in 2012 after received his Diploma in engineering in 2009 from GPTC Cherthala, Alappuzha .He is currently pursuing his M.Tech degree in Applied Electronics at Mahatma Gandhi University Colllege of Engineering, Thodupuzha Under MG University Kottayam. He is working as Lecturer in Govt Polytechnic college, Cherthala, Alappuzha Since June 2012.He was born on 15 th july 1988 at Cherthala.

Mrs.Kavitha.N.Nair received her M.Tech degree from Mahatma Gandhi University College of Engineering, Thodupuzha,Idukki in Applied Electronics. Now she is working as lecturer in ECE Dept. In MGUCE, Thodupuzha 